\title{
Adicción a Facebook y procrastinación académica en estudiantes de la Facultad de Ingeniería y Arquitectura de la Universidad Peruana Unión, Filial Juliaca - 2015.
}

\section{Addiction to Facebook and academic procrastination in students of the Faculty of Engineering and Architecture of Universidad Peruana Unión, Juliaca Branch - 2015}

\author{
Condori Huanca, Yuli Nélida; Mamani Coaquira, Karla Yosimy; Mamani-Cachicatari, Germán \\ E. P. Psicología, Facultad de Ciencias de la Salud, Universidad Peruana Unión
}

Recibido 10 de mayo del 2016-Aceptado 26 de junio del 2016

\begin{abstract}
Resumen
El propósito de la investigación fue describir la relación existente entre adicción a Facebook y procrastinación académica en los estudiantes de la Facultad de Ingeniería y Arquitectura de la Universidad Peruana Unión, Filial Juliaca, la muestra estuvo conformado por 218 estudiantes pertenecientes a dicha facultad, conformado por cuatro Escuelas Profesionales (Ingeniería Civil, Ingeniería Ambiental, Ingeniería de Sistemas e Ingeniería de Industrias Alimentarias). El diseño corresponde al no experimental transaccional, de tipo descriptivo correlacional, se utilizó el Cuestionario de Uso de Facebook, compuesto por una dimensión (Influencia y dependencia del Facebook), que consta de 20 ítems a los que se responde en una escala Likert de 5 opciones; a su vez se utilizó la Escala de Procrastinación Académica (EPA), que consta de 16 ítems, distribuidos en dos dimensiones (autorregulación académica y postergación de actividades). Los resultados evidencian que existe una relación directa en lo que respecta a adicción a Facebook y procrastinación académica; así mismo existe una relación indirecta con la dimensión de autorregulación académica y correlación directa con la dimensión de postergación de actividades.
\end{abstract}

Palabras Clave: Adicción a Facebook, procrastinación, autorregulación, postergación de actividades

\begin{abstract}
The purpose of the research was to describe the relationship between addiction to Facebook and academic procrastination in the students of the Faculty of Engineering and Architecture of the Universidad Peruana Unión, Juliaca Branch, the sample consisted of 218 students belonging to this faculty, conformed by Four Professional Schools (Civil Engineering, Environmental Engineering, Systems Engineering and Food Industry Engineering). The design corresponded to the non-experimental transactional, correlational descriptive type, was used Facebook Questionnaire, composed of a dimension (Influence and dependence of Facebook), consisting of 20 items that are answered on a Likert scale of 5 Options; The Academic Procrastination Scale (EPA), which consists of 16 items, was distributed in two dimensions (academic self-regulation and postponement of activities). The results show that there is a direct relationship with regard to Facebook addiction and academic procrastination; There is also an indirect relationship with the dimension of academic self-regulation and direct correlation with the dimension of postponement of activities.
\end{abstract}

Keywords: Facebook addiction, academic procrastination, self-regulation, postponement of activities

\section{Introducción}

En los últimos años las redes sociales como Hi5, Twitter, WhatsApp o Facebook se han expandido como un fenómeno social, siendo Facebook una de las redes sociales frecuentemente utilizadas. Esta red permite a los usuarios compartir información con personas o grupos de personas a las que se denomina amigos. A pesar de todos los beneficios que las tecnologías de información sociales ofrecen, los adolescentes en la actualidad no utilizan de forma adecuada este medio de comunicación. Es bajo el porcentaje de estudiantes que utilizan el Facebook con fines académicos y una gran mayoría de adolescentes utilizan para fines de diversión y pérdida de tiempo (Josa, 2014).

Correspondencia al autor:

Email: german.mamani@upeu.pe 
El uso adecuado de las tecnologías de la información y comunicación (TICs) están orientadas a facilitar la vida, pero al mismo tiempo el uso no adecuado puede complicarla. En algunas circunstancias, en especial en los adolescentes, estos recursos tecnológicos pueden convertirse en un fin y no en un medio. Si existe un deseo enfermizo por obtener la última novedad tecnológica, las TICs se convierten en un instrumento de placer. El uso de las redes sociales entre ellas el Facebook, puede atrapar a los adolescentes ya que el mundo virtual permite crear en la persona una falsa identidad, distanciarlo o distorsionar el mundo real (Echeburúa \& Corral, 2010).

Los problemas con el uso del internet y las redes sociales surgen cuando las personas descuidan sus rutinas diarias para permanecer conectadas a internet interfiriendo las actividades cotidianas y las horas del sueño nocturno. Algunos usuarios llegan a obviar comidas durante el día. Permanecer conectados a internet y hacer uso de las redes sociales por más de 3 a 4 horas diarias, facilita el aislamiento de la realidad, el desinterés por otros temas, reduce el rendimiento académico, baja el rendimiento laboral, facilita los trastornos de conducta, así como el sedentarismo y la obesidad (Cía, 2013). Todo esto lleva al adolescente a postergar el cumplimiento de sus actividades diarias, fenómeno conocido como procrastinación, que es un trastorno del comportamiento que consiste en postergar de forma sistemática aquellas tareas importantes para el desarrollo de la persona y reemplazarlas por otras más irrelevantes.

En el ámbito académico, la procrastinación consiste en el hábito de posponer las actividades académicas consideradas importantes generando malestar emocional y constituye una práctica frecuente. Más de un 90\% de estudiantes universitarios presentan esta práctica aunque no siempre con carácter crónico (Natividad, 2014). Así mismo Guzmán (2013) menciona que entre $80 \%$ y $95 \%$ de los estudiantes universitarios procrastinan en ocasiones. De éste grupo el $70 \%$ se considera un procrastinador y casi el $50 \%$ procrastina de manera consistente y problemática. Finalmente, como problema crónico afecta de $20 \%$ a $25 \%$ de la población general.

Según una investigación efectuada por el Banco Mundial, en el Perú de cada 100 peruanos, 38 accede al servicio de internet con diferentes fines. Esta cifra se incrementa en niños y adolescentes en edad escolar, que lejos de emplear el internet como una herramienta de aprendizaje se encasillan por muchas horas en el ordenador para acceder a los denominados juegos en red cuyo consumo excesivo encamina a la adicción. Reportes del Ministerio de Salud señalan que en el 2013 se duplicó el número de menores atendidos en hospitales públicos por adicción a los videojuegos o juegos en red (Gómez, Bustincio, \& Espezua, 2014).

Por otro lado, es importante precisar que actualmente los estudiantes tienen la facilidad de manejar un aparato tecnológico como laptop, computadora o celular ya que estos instrumentos facilitan el crecimiento académico, siempre que estos tengan un adecuado uso y se constituyan en su herramienta de trabajo.

El concepto de adicción se asociaba anteriormente solo al abuso de sustancias, drogas y por tanto equivalía a drogodependencia o toxicomanía; en la actualidad el concepto ha cambiado mucho y en poco tiempo. Hoy en día el concepto de adicción se refiere tanto a una sustancia psicoactiva que afecta al organismo como la adicción a una conducta que altera de manera sustancial la vida de la persona afectada. Ambas definiciones de adicción, tanto la adicción a sustancias como las conductas adictivas, se incluyen bajo el concepto genérico de lo que se denomina trastorno adictivo (Cuyún, 2013).

Según la Real Academia Española, una adicción es un hábito por la afición desmedida y de acuerdo con Salamanca (2007), la adicción es la preocupación y uso compulsivo de una experiencia o sustancia sin importar las consecuencias adversas. La adicción generalmente conlleva a una pérdida de control.

La adicción a las nuevas tecnologías se caracteriza por un consumo excesivo de las mismas sean estas el ordenador, internet, móvil, videojuegos o redes sociales. Las adiciones pueden afectar a todas las edades pero suelen darse principalmente en los jóvenes y en los adolescentes. El uso de internet permite comunicarse con otras personas de forma anónima, hablar de temas que cara a cara les sería difícil, expresar emociones, comunicarse virtualmente con personas desconocidas o mantener el contacto con amigos que de otra forma no podrían hacerlo (Arias, Gallego, Rodríguez, \& Del Pozo, 2012)

Merodio (2010) menciona que Facebook es la red social más utilizada, las personas pueden compartir todo tipo de fotos, videos, imágenes, crear un perfil atrayente para conseguir más contactos; asimismo para las empresas es fundamental en ámbitos de publicidad, ya que este sitio permite lanzar ofertas, promociones, anuncios. También existe un espacio para obtener mensajes privados que solo el dueño de la cuenta podrá leer y de este modo mantener contacto con muchas personas conocidas y no conocidas.

La adicción al Facebook es una adicción no convencional a una conducta que al comienzo puede ser tomada como juego (placentero) o como un mecanismo de comunicación, pero con el trascurso del tiempo termina siendo adictiva. El adicto genera una fuerte adicción psicológica hacia ellas, actuando de forma impetuosa y perdiendo inclinación por otras actividades que anteriormente habían sido gratificantes. 
Según el diccionario de la Real Academia de la Lengua Española define la palabra "procrastinar" como "diferir, aplazar". Según el Online Etymology Dictionary, el término proviene del latín y se compone de las palabras pro, que significa "adelante"/ "a favor de" y crastinus, que significa "del mañana". Sin embargo, las implicaciones del concepto desde el punto de vista psicológico son bastante más complejas. El diccionario inglés Oxford Dictionary of English define la palabra "procrastinar" como "posponer una acción, especialmente sin una buena razón”, añadiendo un nuevo matiz a la idea.

Según Lay (1986), la procrastinación también implica que la acción que está siendo aplazada es necesaria o importante para el individuo, este aplazamiento es voluntario y no impuesto desde fuera, en consecuencia la persona en cuestión procrastina a pesar de ser consciente de las consecuencias negativas que puede tener el hecho de aplazar la acción (Steel, 2007).

Según Pardo, Perilla y Salinas (2014), la procrastinación académica se refiere a la evitación voluntaria de responsabilidades y actividades, causada porque los estudiantes realizan las tareas dentro de un tiempo determinado, pero no poseen motivación a la hora de ejecutarlas, por percibir estas obligaciones como aversivas

Por lo mencionado, se pretende determinar la relación entre adicción a Facebook y procrastinacion académica en estudiantes universitarios de la Facultad de Ingeniería y Arquitectura.

\section{Método}

El estudio corresponde al tipo de investigación correlacional, se utiliza cuando se tiene como propósito conocer la relación existente entre dos o más conceptos, categorías o variables en un contexto en particular. Asocia variables mediante un patrón predecible para un grupo de pobladores. Ofrecen predicciones, explican la relación entre variables y cuantifican relaciones entre variables (Hernandez, Fernández, \& Baptista, 2010).

Según Hernández et al. (2010) y Valderrama (2002) el diseño de la investigación es no experimental, transaccional o transversal, ya que no existe manipulación activa de alguna variable y se busca establecer la relación de variables medidas en una muestra en un único momento del tiempo.

La población de estudio comprende el total de 1002 estudiantes de la Facultad de Ingeniería, conformado por las Escuelas Profesionales de Ingeniería Civil, Ingeniería Ambiental, Ingeniería de Sistemas e Ingeniería de Industrias Alimentarias de la Universidad Peruana Unión Filial Juliaca.

Para la determinación de la muestra, se realizó un muestreo probabilístico estratificado, quedando la muestra constituida por 218 estudiantes (122 de Ing. Civil, 48 de Ing. Ambiental, 30 de Ing. de Sistemas y 18 estudiantes de Ing. Alimentos)

Según Valderrama (2002) se utilizó la técnica de la encuesta, los datos fueron obtenidos mediante la aplicación de dos inventarios de adicción a Facebook y procrastinación académica.

Luego de la aplicación, se realizó el trabajo estadístico, procesando los datos, para esto se utilizó el paquete estadístico SPSS versión 22 (The Pactage Sattistical For The Social Sciencies). Para el análisis de los datos se utilizó el estadístico de correlación de Pearson. Los resultados fueron procesados en tablas estadísticas de doble entrada para comprobar la relación entre las variables.

\section{Resultados}

La tabla 1 muestra los niveles de adicción al Facebook que presentan los estudiantes de la Facultad de Ingeniería y Arquitectura de la Universidad Peruana Unión, Filial Juliaca, en el cual se aprecia que el 87.6\% de los estudiantes presentan un nivel de adicción moderada, el $11.5 \%$ de los estudiantes presentan un nivel de adicción leve, indicando que los estudiantes son tendientes a presentar comportamientos adictivos y sólo un $0.9 \%$ presentan una adicción severa. Ningún estudiante presenta un nivel de no adicción.

Tabla 1

Nivel de Adicción de Facebook que presentan los estudiantes de la Facultad de Ingeniería y Arquitectura de la Universidad Peruana Unión, Filial Juliaca - 2015.

\begin{tabular}{lccc}
\hline & Frecuencia & Porcentaje & Porcentaje acumulado \\
\hline Adicción leve & 25 & 11,5 & 11,5 \\
Adicción moderada & 191 & 87,6 & 99,1 \\
Adicción severa & 2 &, 9 & 100,0 \\
Total & 218 & 100,0 & \\
\hline
\end{tabular}


Con respecto al nivel de Procrastinación Académica, la tabla 2 muestra los niveles que presentan los estudiantes de la Facultad de Ingeniería y Arquitectura de la Universidad Peruana Unión, Filial Juliaca, y se aprecia que el 45.4\% de los estudiantes presentan un nivel promedio, el $33.9 \%$ presenta un nivel alto, indicando que los estudiantes no presentan conductas autorreguladoras y de responsabilidad académica; el $10.1 \%$ y $1.8 \%$ de los estudiantes presentan un nivel bajo y muy bajo respectivamente, indicando que este grupo de estudiantes poseen la capacidad de autorregulación y responsabilidad académica; así mismo el $8.7 \%$ de los estudiantes presentan un nivel muy alto de procrastinación académica.

Tabla 2

Nivel de Procrastinación Académica que presentan los estudiantes de la Facultad de Ingeniería y Arquitectura de la Universidad Peruana Unión, Filial Juliaca - 2015.

\begin{tabular}{lcc}
\hline \multicolumn{1}{c}{ Nivel de procastinación } & Frecuencia & Porcentaje \\
\hline Muy bajo & 4 & 1,8 \\
Bajo & 22 & 10,1 \\
Promedio & 99 & 45,4 \\
Alto & 74 & 33,9 \\
Muy alto & 19 & 8,7 \\
Total & 218 & 100,0 \\
\hline
\end{tabular}

Con respecto al nivel de Autorregulación Académica, la tabla 3 muestra los niveles que presentan los estudiantes de la Facultad de Ingeniería y Arquitectura de la Universidad Peruana Unión, Filial Juliaca, y se aprecia que el 61.9\% de los estudiantes presentan un nivel promedio, el 35.8\% presenta un nivel alto, indicando que los estudiantes presentan conductas autorreguladoras y de responsabilidad académica. El $2.3 \%$ presenta un nivel bajo, indicando que este grupo de estudiantes no poseen la capacidad de autorregulación y responsabilidad académica los cuales están propensos a la procastinación.

Tabla 3

Nivel de Autorregulación Académica que presentan los estudiantes de la Facultad de Ingeniería y Arquitectura de la Universidad Peruana Unión, Filial Juliaca - 2015.

\begin{tabular}{ccc}
\hline Nivel de autorregulación & Frecuencia & Porcentaje \\
\hline Bajo & 5 & 2,3 \\
Promedio & 135 & 61,9 \\
Alto & 78 & 35,8 \\
Total & 218 & 100,0 \\
\hline
\end{tabular}

Con respecto al nivel de postergación de actividades, la tabla 4 muestra los niveles de postergación y se aprecia que el $57.8 \%$ de los estudiantes presentan un nivel promedio, el $32.6 \%$ presenta un nivel alto, indicando que los estudiantes postergan sus actividades académicas, no presentan conductas de responsabilidad académica. El $8.3 \%$ de los estudiantes presentan un nivel bajo, indicando que este grupo de estudiantes poseen responsabilidad académica.

Tabla 4

Nivel de postergación de actividades que presentan los estudiantes de la Facultad de Ingeniería y Arquitectura de la Universidad Peruana Unión, Filial Juliaca - 2015.

\begin{tabular}{|c|c|c|}
\hline & Frecuencia & Porcentaje \\
\hline Bajo & 18 & 8,3 \\
\hline Promedio & 126 & 57,8 \\
\hline Alto & 71 & 32,6 \\
\hline Muy alto & 3 & 1,4 \\
\hline Total & 218 & 100,0 \\
\hline
\end{tabular}

El análisis de correlación determina la existencia de una relación directa y significativa entre adicción al Facebook y Procrastinación Académica que presentan los estudiantes. En la tabla 5 Se aprecia un coeficiente de correlación r= 696; una correlación indirecta y significativa entre adicción al Facebook y Autorregulación Académica ( $\mathrm{r}=$-.632), y una correlación directa y significativa entre adicción al Facebook y postergación de actividades (r= .439) (Tabla 5) 
Tabla 5

Análisis de correlación entre Adicción a Facebook y procrastinación académica (postergación de actividades, autorregulación de actividades) en estudiantes de la Facultad de Ingeniería y Arquitectura.

\begin{tabular}{|c|c|c|c|c|c|}
\hline & & $\begin{array}{l}\text { Adicción al } \\
\text { Facebook }\end{array}$ & $\begin{array}{l}\text { Procrastinación } \\
\text { Académica }\end{array}$ & $\begin{array}{l}\text { postergación de } \\
\text { actividades }\end{array}$ & $\begin{array}{l}\text { Autorregulación } \\
\text { Académica }\end{array}$ \\
\hline \multirow[t]{3}{*}{ Adicción al Facebook } & Correlación de Pearson & 1 &, $696(* *)$ &, $439(* *)$ &,$- 632(* *)$ \\
\hline & Sig. (bilateral) & &, 000 & 000 & 000 \\
\hline & $\mathrm{N}$ & 218 & 218 & 218 & 218 \\
\hline \multirow[t]{3}{*}{$\begin{array}{l}\text { Procrastinación } \\
\text { Académica }\end{array}$} & Correlación de Pearson &, $696(* *)$ & 1 &, $598(* *)$ &,$- 924(* *)$ \\
\hline & Sig. (bilateral) &, 000 & & ,000 & 000 \\
\hline & $\mathrm{N}$ & 218 & 218 & 218 & 218 \\
\hline \multirow[t]{3}{*}{$\begin{array}{l}\text { postergación de } \\
\text { actividades }\end{array}$} & Correlación de Pearson &, $439(* *)$ &, $598(* *)$ & 1 &,$- 248(* *)$ \\
\hline & Sig. (bilateral) & ,000 & ,000 & & 000 \\
\hline & $\mathrm{N}$ & 218 & 218 & 218 & 218 \\
\hline \multirow[t]{3}{*}{$\begin{array}{l}\text { Autorregulación } \\
\text { Académica }\end{array}$} & Correlación de Pearson &,$- 632(* *)$ &,$- 924(* *)$ &,$- 248(* *)$ & 1 \\
\hline & Sig. (bilateral) &, 000 & ,000 &, 000 & \\
\hline & $\mathrm{N}$ & 218 & 218 & 218 & 218 \\
\hline
\end{tabular}

** La correlación es significativa al nivel 0,01 (bilateral).

\section{Discusión y conclusiones}

Los resultados muestran que el $87.1 \%$ evidencian un nivel de adicción al Facebook moderado, indicándonos que los estudiantes tienden a presentar comportamientos adictivos. Sigue el nivel leve con un $11.5 \%$. Un $0,9 \%$ correspondiente a 2 estudiantes presentan un nivel de adicción severa. Estos resultados guardan relación con los reportados por Villavicencio (2015) quien menciona que el mayor porcentaje de estudiantes presentan un nivel medio de adicción a Facebook (56.0\%), pero difiere en el porcentaje de estudiantes con un nivel alto de adicción. Mientras que el $24 \%$ de los adolescentes del colegio Internacional Rudolf Steiner presentan adicción severa, en los estudiantes de la Universidad Peruana Unión sólo el 0.9\% presentan un nivel de adicción severa. Esto podría ser explicado por la cultura organizacional de la Universidad Peruana Unión, donde la formación del estudiante es acompañada por un programa de tutoría universitaria, dicho programa contempla el trabajo en 4 áreas: tutoría académica, psicológica, física y espiritual.

Muchos de los estudiantes de la Universidad Peruana Unión vienen utilizando el Facebook como un medio de comunicación grupal, es decir que las comunicaciones académicas del salón lo realizan a través de una cuenta del Facebook. Esta práctica se ve incrementado en los estudiantes de la Facultad de Ingeniería, en especial de la carrera de Ingeniería de Sistemas, por estar ligado al uso de tecnologías de información.

Por lo anteriormente mencionado, puede que el empleo excesivo de las redes sociales provoque en los adolescentes ciertos niveles de dependencia, la misma que varía entre baja, media y alta. Por lo tanto, la exposición prolongada de los adolescentes a internet y de manera específica a la red social Facebook, puede provocar en los mismos niveles elevados de dependencia e inclusive adicción.

Con respecto a la procrastinación académica un $45.4 \%$ se encuentra en un nivel promedio, seguido del nivel alto y muy alto en un $33.9 \%$ y $8.7 \%$ respectivamente, indicando que este grupo de estudiantes postergan sus actividades académicas por otras actividades menos importantes, y solamente el $11.9 \%$ de los estudiantes presentan un nivel bajo y muy bajo. Álvarez (2010) reportó que no existen diferencias significativas en la procrastinación según género y grado de estudios. Carranza y Ramírez (2013), reportaron que el 15.9\% de estudiantes universitarios de Tarapoto presentan un nivel alto de procrastinación, guardando similitud a los encontrados en la presente investigación.

Los resultados realizados por Balkis y Duru (citado en Pardo et al., 2014), indican que, en estudiantes de psicología, el $23 \%$ de la población presenta una procrastinación alta y el $27 \%$ presenta una procrastinación moderada. Con respecto a la entrega de trabajos, la mayoría de los participantes afirmó que estudiaba un día antes del examen; la minoría afirmó que estudiaba dos semanas antes para la entrega de trabajos. Además, el estudio también evidencia que hay una correlación negativa entre la edad y el comportamiento de procrastinación académica, puesto que a mayor edad se procrastina menos.

Respecto a la autorregulación académica $35.8 \%$ de los estudiantes presentan una autorregulación académica alta, indicando que este grupo presenta conductas autorreguladoras y de responsabilidad académica, el $61.9 \%$ presenta un nivel promedio de autorregulación y el $2.3 \%$ presenta un nivel bajo de autorregulación, indicando que los estudiantes no presentan responsabilidad 
académica. Así mismo se aprecia que el $32.6 \%$ de los estudiantes presentan un nivel alto de postergación de actividades académicas. Estos resultados guardan relación con los reportados por Carranza y Ramírez (2013) quienes mencionan que la mayoría de los estudiantes de la Facultad de Ingeniería y arquitectura presentan un nivel alto de procrastinación (55.4\%).

Los resultados muestran que existe una correlación directa y significativa entre la adicción al Facebook y procrastinación, así como con sus dimensiones de procrastinación (postergación de actividades y Autorregulación Académica)

\section{Referencia}

Álvarez, Ó. R. (2010). Procrastinación general y académica en una muestra de estudiantes de secundaria de Lima Metropolitana. Persona, 159-177.

Arias, O., Gallego, V., Rodríguez, M. J., \& Del Pozo, M. Á. (2012). Adicción a las nuevas tecnologías. Psicología de las Adicciones, I, 2-6.

Carranza, R., \& Ramírez, A. (2013). Procrastinación y características demográficas asociados en estudiantes universitarios. Apuntes Universitarios, III(2), 95-108.

Cía, A. (2013). Las adicciones no relacionadas a sustancias (DSM-5, APA, 2013): un primer paso hacia la inclusión de las Adicciones Conductuales en las clasificaciones categoriales vigentes. Revista de Neuro-Psiquiatría, 76(4), $210-217$. Recuperado de http://www.redalyc.org/articulo.oa?id=372036946004

Cuyún, M. I. (2013). Adicción a redes sociales en jóvenes (Estudio realizado con jóvenes de 14-16 años del Colegio Liceo San Luis de Quetzaltenango). Tesis para optar el Título de Psicóloga Clínica, Universidad Rafael Landívar, Facultad de Humanidades.

Echeburúa, E., \& Corral, P. (2010). Adicción a las nuevas tecnologías y a las redes sociales en jóvenes: un nuevo reto. Adicciones, 22(2), 91-96. Recuperado de

http://www.ehu.eus/echeburua/pdfs/Adicci\%C3\%B3n\%20a\%20las\%20redes\%20sociales.pdf

Gómez, J., Bustincio, M., \& Espezua, A. (30 de Marzo de 2014). Internet: ¿Distracción sana o nociva para escolares? Los Andes. Obtenido de http://losandes.com.pe/Sociedad/20140330/79310.html

Guzmán, D. (2013). Procrastinación. Una mirada clínica. Barcelona. Recuperado de https://drive.google.com/a/upeu.pe/file/d/0B_Rzkq9hb1dfWWxZTWowNmYwbnc/view

Hernandez, R., Fernández, C., \& Baptista, P. (2010). Metodología de la investigación (Quinta ed.). México: McGraw-Hill.

Josa, R. a. (2014). El efecto Facebook y sus repercusiones psicológicas y pedagógicas en los estudiantes de los novenos años del Colegio Universitario UTN en el periodo académico 2013-2014. trabajo de Grado previo a la obtención del título de Licenciada en Ciencias de la Educación en la especialidad de Psicología Educativa y Orientación Vocacional, Universidad Técnica del Norte, Facultad de Educación, Ciencia y Tecnología, Ibarra. Recuperado de http://repositorio.utn.edu.ec/bitstream/123456789/4203/1/05\%20FECYT\%202103\%20TESIS.pdf

Lay, C. (1986). At last, my research article on procrastination. Journal of Research in Personality(20), 474-495.

Merodio, J. (2010). Como empezar a promocionar tú negocio en redes sociales (Primera ed.). México D.F.

Natividad, L. A. (2014). Análisis de la procrastinación en estudiantes universitarios. Tesis Doctoral, Universidad de Valencia, Facultad de Psicología, Valencia, España.

Pardo, D., Perilla, L., \& Salinas, C. (2014). Relación entre procrastinación académica y ansiedad-rasgo en estudiantes de psicología. Cuadernos Hispanoamericanos de Psicología, 14(1), 31-44.

Steel, P. (2007). The nature of procrastination: A meta-analytic and theoretical review of quintessential self-regulatory failure. Psychological Bulletin, 65-94.

Valderrama, S. (2002). Pasos para elaborar proyectos y tesis de investigación científica. Lima: San Marcos.

Villavicencio, N. (2015). Adicción a Facebook en adolescentes de quinto curso del Colegio Internacional Rudolf Steiner. Trabajo de fin de carrera para obtener el Título de Psicopedagóga, Universidad Internacional Sek, Facultad de Psicología, Quito, Ecuador. 